\title{
Chapter 16. Management of CKD
}

(C) Japanese Society of Nephrology 2009

- The goal of CKD management is to suppress both the progression to end-stage kidney disease (ESKD) and the occurrence of cardiovascular disease (CVD).

- A multi-modal therapeutic approach is essential for the suppression of ESKD and CVD development.

\section{The purpose of CKD management}

- The primary aim of CKD management is to prevent CKD or retard its progression to ESKD, which severely impairs the quality of life of CKD patients.

- The second aim is to suppress newly onset CVD or the progression of preexisting CVD through management of CKD, which itself is a risk factor for CVD development.

- The management of ESKD requires relatively costly renal replacement therapies, such as hemodialysis, peritoneal dialysis, or kidney transplantation. Therefore, the management of CKD is critical for maintaining an economically viable public healthcare system.

\section{Multi-modal therapy to suppress the development of ESKD and CVD}

- The development of ESKD and CVD can be suppressed by multi-modal therapy that help interrupt a vicious cycle of the disease. Targets for interventions are shown in Fig. 16-1.

- Modification of lifestyles (refer to "treatment of hypertension"). Weight control and stopping with smoking are essential parts of anti-hypertension therapy. Modification of lifestyle may suppress atherosclerosis, which will result in retarding CKD progression (a).
- Diet therapy (refer to "Principle of diet therapy of CKD"). Salt restriction is essential as an anti-hypertension therapy. Restriction of dietary protein depending on the CKD stage is assumed to inhibit CKD progression (b).

- Treatment of hypertension (refer to "Antihypertensive therapy"). Breakage of a vicious cycle caused by both CKD and hypertension entails strict antihypertensive therapy. Angiotensin converting enzyme (ACE) inhibitors and angiotensin-receptor blockers (ARBs) play a central part in the therapy, but the co-administration of other antihypertensive agents is also necessary for an optimal blood pressure to be achieved (c).

- Reduction of proteinuria and microalbuminuria. Reduction of urinary protein or microalbumin generally follows lowered blood pressure induced by ACE inhibitor or ARB therapy. The majority of their inhibitory effects on CKD progression rely upon a reduction of urinary protein. Other options include antiplatelet agents and similar drugs which can also suppress the urinary protein level. The goal of urinary protein excretion should $<0.5 \mathrm{~g} / \mathrm{g}$ creatinine (d).

- Treatment of dyslipidemia. Dyslipidemia may be a potential promoter of CKD progression by various mechanisms and is one of the most significant risk factors for CVD. Hence, management of dyslipidemia in CKD is indispensable for suppressing the progression to both ESKD and CVD (e).

- Treatment of diabetes and glucose intolerance. Strict treatment of diabetes is essential for the suppression of ESKD or the development of CVD (f).

- Treatment of anemia. Renal anemia appears with progressing CKD stage. Anemia is not only a risk factor for CKD progression but also for CVD. Its 
Fig. 16-1 Targets for interventions in preventing endstage kidney disease (ESKD) and cardiovascular disease $(C V D)$. DM Diabetes mellitus, $I G T$ impaired glucose tolerance, $C K D$ chronic kidney disease

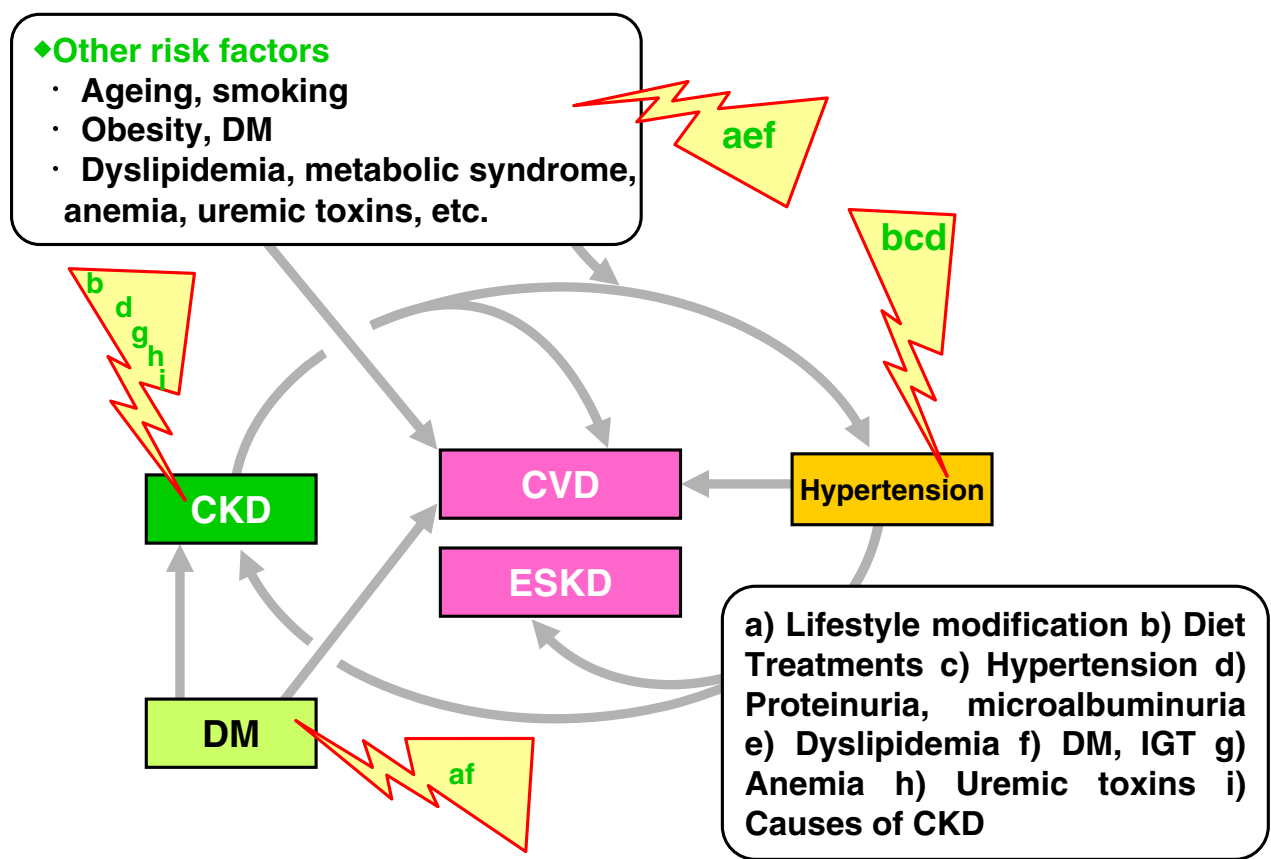

treatment is therefore critical for the suppression of both ESKD and CVD (g).

- Treatment of uremic toxins. An oral adsorbent may be expected to improve uremic symptoms (h).
- Treatment of an underlying disease of CKD. If a causative disease for CKD is determined, its treatment is primarily recommended (i). 